\title{
A Novel Algorithm Based on 2-Additive Measure and Shapley Value and Its Application in Land Pollution Remediation
}

\author{
Jian Lin $\mathbb{D},{ }^{1,2}$ Qiongling Wu, ${ }^{1}$ Riqing Chen $\mathbb{D}^{2},{ }^{2}$ and Zhiyong Tian ${ }^{3}$ \\ ${ }^{1}$ The Digital Fujian Research Institute of Big Data for Agriculture and Forestry, Fujian Agriculture and Forestry University, \\ Fuzhou 350002, China \\ ${ }^{2}$ Key Laboratory of Smart Agriculture and Forestry, Fujian Agriculture and Forestry University, Fuzhou 350002, China \\ ${ }^{3}$ Beijing Intelligent Logistics System Collaborative Innovation Center, Beijing Wuzi University, Beijing 101149, China \\ Correspondence should be addressed to Jian Lin; linjian36@163.com and Riqing Chen; riqing.chen@fafu.edu.cn
}

Received 11 October 2019; Revised 15 March 2020; Accepted 10 April 2020; Published 5 May 2020

Academic Editor: Juan P. Amezquita-Sanchez

Copyright ( 2020 Jian Lin et al. This is an open access article distributed under the Creative Commons Attribution License, which permits unrestricted use, distribution, and reproduction in any medium, provided the original work is properly cited.

\begin{abstract}
In this article, a new aggregation operator called the Young-Shapley optimal weight (Y-SOW) operator is proposed to aggregate heterogeneous information in group decision-making. The Y-SOW operator combines the Shapley value with the Young inequality. Meanwhile, a series of special cases and main properties of the Y-SOW operator are studied. Furthermore, the dispersion maximization model of the Y-SOW operator is established to obtain the optimal 2-additive measure. In the Shapley value method of the cooperative game, the 2-additive measure not only simplifies the complexity of fuzzy measures but also solves the interaction between attributes. The Shapley value of the 2-additive measure is explored to the weight of the Y-SOW operator. Finally, the Y-SOW operator-based multiattribute group decision (YSMGAD) algorithm is proposed. The application of the YSMGAD algorithm for land pollution remediation is analyzed.
\end{abstract}

\section{Introduction}

Group decision-making is rapidly developed to an important branch of modern decision sciences, which helps to gather the wisdom of experts from different fields. The transition from individual to group decision-making is a major step forward to cope with increasingly complex human activities. To this end, group decision-making has been recognized and used in economic, military, agricultural, and other fields [1-6].

In the state-of-art literature, many methods of aggregation operators and their weight determination have been proposed for multiattribute decision-making. Yager [7] introduced an ordered weighted averaging (OWA) operator, where the input arguments were rearranged in the descending order, and the weight vector was only related to its ordered position. Xu and $\mathrm{Da}[8,9]$ developed the ordered weighted geometric averaging (OWGA) operator for multiattribute decision-making. Chen and Liu [10] proposed an extension of the OWA operator called an ordered weighted harmonic mean (OWHA) operator. In 2004, Yager [11] used a generalized mean in the OWA operator and a generalized ordered weighted averaging (GOWA) operator. Based on a minimizing model, Zhou and Chen presented the generalized ordered weighted logarithm averaging (GOWLA) operator [12], generalized ordered weighted harmonic averaging (GOWHA) operator [13], and so on. Merigo et al. expanded the OWA operator and proposed the ordered weighted averaging-weighted average (OWAWA) operator [14], which unified the OWA operator in the same formulation. Other generalizations of the aggregation operators were observed in $[12,15-21]$. The operators proposed above only deal with additive or multiplicative information alone. The existing literature is not sufficient to solve the problem when two kinds of information appear simultaneously in group decision-making.

In 1995, Grabisch [22-25] proposed the fuzzy measure as an aggregation operator for multiattribute decision-making. However, the fuzzy measure requires a large number of parameters, which is difficult to implement. In order to 
reduce the computational complexity, various forms and methods of determining the fuzzy measure have been proposed. The choquet integral [26-28] is a nonlinear integration operator defined on the basis of fuzzy measures, which can effectively deal with the interaction between decision attributes. The premise of the integral is utilized to determine the fuzzy measure, which is complicated. If there are $n$ attributes, $2^{n}-2$ parameters are needed. Sugeno [29] proposed a $\lambda$ measure that only requires $n$ parameters but cannot fully describe the interaction between attributes. In 1996, Grabisch [30] proposed the $k$-additive measure. The Shapley value of a single attribute and the interaction among $k$ attributes were determined, the computational complexity was reduced, and the representing ability between the attributes was improved to some extent.

In reality, the attribute value often includes both additive and multiplicative information; however, it is difficult to aggregate precisely in one matrix. Based on the Young inequality and Shapley value, a new optimal operator called the Young-Shapley optimal weight (Y-SOW) operator is proposed. Meanwhile, a series of special cases and the main properties of the Y-SOW operator are studied. Because one certain interaction exists between the attributes, the 2-additive measure is introduced to reduce the complexity of the fuzzy measure. The Shapley value method is the most effective and widely used method in cooperative games. Therefore, the dispersion maximization model based on the 2 -additive measure and the Shapley value is established to obtain the optimal 2-additive measure. Some formula and programming models are also provided to effectively determine the 2-additive measure. The Shapley value of the 2additive measure is used as the weight of the Y-SOW operator. Finally, the Y-SOW operator-based multiattribute group decision (YSMAGD) algorithm is proposed to effectively aggregate the heterogeneous data. However, the Y-SOW operator can only aggregate the real-type data and has certain limitations for the other types of data, such as interval-type data.

The rest of this article is organized as follows. In Section 2 , the basic concepts of common aggregation operators, fuzzy measures, and Shapley values are reviewed. In Section 3 , the common Y-SOW operator is proposed, and some special cases and ideal characteristics of the Y-SOW operator are also proved. In Section 4, the Y-SOW operator-based multiattribute group decision (YSMAGD) algorithm is established. In Section 5, the YSMAGD algorithm is applied to sequence the remediable location of land pollution. In Section 6, summary is given.

\section{Preliminaries}

2.1. Several Commonly Used Information Aggregation Operators. The ordered weighted averaging (OWA) operator was proposed by Yager [7] in 1988 and is widely used in a series of decision problems, as defined below.

Definition 1. Let $R$ be the set of real number. AnOWA operator is a mapping, OWA: $R^{n} \longrightarrow R$ is satisfied:

$$
\operatorname{OWA}\left(a_{1}, \ldots, a_{n}\right)=\sum_{j=1}^{n} w_{j} b_{j},
$$

then OWAis called an ordered weighted averaging operator, where $b_{j}$ is the $j$ th largest of the arguments $a_{1}, \ldots, a_{n}$ and the weight vector $W=\left(w_{1}, \ldots, w_{n}\right)^{T}$ satisfies $\sum_{j=1}^{n} w_{j}=1$, $w_{j} \in[0,1]$ and $(j=1,2, \ldots, n)$.

The OWA operator is an effective aggregation method that rearranges the arguments and then weights it according to the sequence position to weaken the adverse effects of the extreme value. It is characterized by considering only the positional relationship of the arguments in the ordering process. The OWA operator has many desirable properties such as monotonicity, boundedness, idempotency, and permutation invariance. When $b_{j}=a_{j}$, holds for all $j=1,2, \ldots, n$, then the OWA operator becomes a weighted averaging (WA) operator [31].

Yager also proposed the BUM function $Q$ [32] to calculate the associated weight of the OWA operator, which satisfies $Q(0)=0, Q(1)=1$, and $Q(x) \leq Q(y)$ for any $0 \leq x \leq y \leq 1$, that is,

$$
w_{j}=Q\left(\frac{j}{n}\right)-Q\left(\frac{j-1}{n}\right), \quad j=1,2, \ldots n .
$$

The BUM function is called the fuzzy semantic quantization operator, and the expression can be stated as follows:

$$
Q(x)= \begin{cases}0, & x \leq x_{1}, \\ \frac{x-x_{1}}{x_{2}-x_{1}}, & x_{1} \leq x \leq x_{2}, \\ 1, & x \geq x_{2},\end{cases}
$$

where $x_{1}, x_{2}$, and $x$ are in the range of $[0,1]$. When we choose the pair $(0,0.5),(0.3,0.8)$, and $(0.5,1)$, the fuzzy linguistic representation is "at least half," "more," and "as many as possible," respectively.

Definition 2. An OWG operator [33] is a mapping, OWG: $I^{n} \longrightarrow I$ and $I=\{x \mid x \geq 0\}$, according to the following formula:

$$
\operatorname{OWG}\left(a_{1}, \ldots, a_{n}\right)=\prod_{j=1}^{n} b_{j}^{w_{j}},
$$

then OWG is called an ordered weighted geometric operator, where $b_{j}$ is the $j$ th largest of the arguments $a_{1}, \ldots, a_{n}$, and the weight vector $w=\left(w_{1}, \ldots, w_{n}\right)^{T}$ satisfies $\sum_{j=1}^{n} w_{j}=$ 1 and $w_{j} \in[0,1], j=1,2, \ldots, n$.

The generalized weighted averaging (GWA) operator was first presented by Yager [11] based on the generalized mean. Assuming that the fusion result is an $\mathrm{n}$-dimensional function $f$, we can construct a penalty function $J=\sum_{j=1}^{n} w_{j}\left(f^{\theta}-a_{j}^{\theta}\right)^{2}$ and the minimization problem:

$$
\min J=\sum_{j=1}^{n} w_{j}\left(f^{\theta}-a_{j}^{\theta}\right)^{2} .
$$


Let $(\partial J / \partial f)=0$, and the aggregation method for obtaining the GWA operator is shown as follows:

$$
\operatorname{GWA}\left(a_{1}, \ldots, a_{n}\right)=\left(\sum_{j=1}^{n} w_{j} a_{j}^{\theta}\right)^{1 / \theta} \text {. }
$$

If the arguments $a_{1}, \ldots, a_{n}$ in the GWA operator are arranged in a descending order, the generalized ordered weighted averaging (GOWA) operator can be obtained. The GWA operator also has many desired properties, such as monotonicity, boundedness, idempotency, and permutation invariance. When $\theta=1, \theta=-1$, and $\theta \longrightarrow 0$, then the GWA operator reduces to the weighted averaging (WA) operator, the weighted harmonic averaging (WHA) operator, and the weighted geometric averaging (WGA) operator, respectively.

2.2. Fuzzy Measure and Shapley Value. The aggregation operators used in the traditional multiattribute decisionmaking are generally based on the premise that the attributes are independent and do not interact with each other, but the actual situations are often interactive or dependent. To deal with the abovementioned phenomenon, the fuzzy measure $[12,20-27]$ is introduced as follows.

Definition 3 (see [29]). Let $N$ be the attribute set and $P(N)$ be the power set of $N$. If set function $\mu: P(N) \longrightarrow[0,1]$ satisfies the following conditions:

(i) $\mu(\phi)=0, \mu(N)=1$

(ii) $\forall A, s B \in P(N), A \subseteq B \Rightarrow \mu(A) \leq \mu(B)$
Then, $\mu$ is a fuzzy measure on $P(N)$.

Fuzzy measure is difficult to calculate and requires a large number of parameters. Grabisch proposed the 2-additive fuzzy measure based on pseudo-Boolean function and Mobius transform [29].

Definition 4. Let $f:\{0,1\}^{n} \longrightarrow R$ be a pseudo-Boolean function [26]. Where $\{0,1\}^{n}$ denotes the entirety of all n-dimensional Boolean vectors. Let $X=\left\{x_{1}, \ldots, x_{n}\right\}$, $\forall A \subseteq N$. Any fuzzy measure $\mu$ can be seen as a particular case of pseudo-Boolean function denoted by

$$
\mu(A)=\sum_{T \subseteq N}\left[a_{T} \prod_{i \in T} y_{i}\right]
$$

where $\quad a_{T} \in R, \quad y=\left(y_{1}, y_{2}, \ldots, y_{n}\right) \in\{0,1\}^{n}, \quad$ and $a_{T}=\sum_{S \subseteq T}(-1)^{t-s} \mu(S)$ is called the Mobius transform coefficient. Obviously, $y_{i}=1$ if and only if $i \in A$. The fuzzy measure $\mu$ defined on $(X, P(N))$ is a k-additive fuzzy measure, and the corresponding pseudo-Boolean function is a k-order linear polynomial, that is, $\forall T \in N$, if $|T|>k$, then $a_{T}=0$ and $\exists T_{0} \in P(N),\left|T_{0}\right|=k \Rightarrow a_{T_{0}}=0$.

From Definition 4, we get that 1-additive measures are additive measures and n-additive measures are fuzzy measures. Especially, when $k=2$, by equation (7) we get a 2additive measure $\mu$.

Definition 5 (see [30]). For a 2-additive measure $\mu$, $\forall S \subseteq N, s \geq 2$, then

$$
\mu(S)=\sum_{i=1}^{n} a_{i} x_{i}+\sum_{\{i, j\} \subseteq N} a_{i j} x_{i} x_{j}=\sum_{i \in S} a_{i}+\sum_{\{i, j\} \subseteq S} a_{i j}=\sum_{\{i, j\} \subseteq S} \mu(i, j)-(s-2) \sum_{i \in S} \mu(i),
$$

where $\mu(i)=a_{i}, \mu(i, j)=a_{i}+a_{j}+a_{i j}$

It is well known that a 2 -additive measure requires only $n(n+1) / 2$ parameters, and it will be simpler to solve a 2 additive measure than to solve a usual fuzzy measure.

Definition 6 (see [30]). Let $\mu$ be the fuzzy measure on $N, N=\{1, \ldots, n\} . \quad \forall i, j \in N \exists \mu(i), \mu(i, j)$, the 2-additive fuzzy measure satisfies the following conditions:

(i) $\mu(i) \geq 0, \forall i \in N$,

(ii) $\sum_{\{i, j\} \subseteq N} \mu(i, j)-(n-2) \sum_{i \in N} \mu(i)=1$,

(iii) $\sum_{i \subseteq S \backslash k}(\mu(i, k)-\mu(i)) \geq(s-2) \mu(k), \forall S \in N, s \geq 2$,

where $s$ and $n$ are the cardinalities of Sand $N$, respectively.

The Shapley value [34] is determined in a grand coalition $N$ based on the marginal contribution of players to obtain the optimal benefit distribution. In order to avoid the irrationality of the average distribution and show certain rationality and fairness, the Shapley value method is widely used for cooperative games.
Definition 7. Vector $\operatorname{Sh}(N, \mu)=\left(\operatorname{Sh}_{1}(N, \mu), \operatorname{Sh}_{2}(N, \mu) \ldots\right.$, $\left.\mathrm{Sh}_{n}(N, \mu)\right)$, and its components are defined by

$$
\begin{aligned}
& \operatorname{Sh}_{i}(N, \mu) \\
& \quad=\sum_{T: i \in T \subseteq N} \frac{(|T|-1) !(|N|-|T|) !}{|N| !}[\mu(T)-\mu(T \backslash\{i\})], \quad \forall i \in N,
\end{aligned}
$$

where $\mu$ is a fuzzy measure on $N$ and $|N|$ and $|T|$ are the cardinality of set $N$ and $T$ respectively.

Let $\mu$ be a superadditive fuzzy measure on $N$, and it follows that $\mu(T) \geq \mu(T \backslash\{i\})$ for all $\forall i \in T \subseteq N$. Obviously, we have

$$
\begin{aligned}
& \text { (i) } \sum_{i=1}^{n} \operatorname{Sh}_{i}(N, \mu)=1, \\
& \text { (ii) } \operatorname{Sh}_{i}(N, \mu) \geq 0(i=1, \ldots, n) .
\end{aligned}
$$

It means that $\operatorname{Sh}(N, \mu)$ can be viewed as a weight vector here, named as the Shapley weight vector. 


\section{The Young-Shapley Optimal Weight (Y-SOW) Operator}

\subsection{The Proposed Operator and Its Equivalent Expression}

Lemma 1 (Young inequality, see [35]). $\forall a, b \geq 0, \lambda \in[0,1]$, it holds that

$$
a^{\lambda} b^{1-\lambda} \leq \lambda a+(1-\lambda) b
$$

if and only if $a=b$, and the equation holds.
In the aggregation process, let $a_{1}, \ldots, a_{n}$ be the nonnegative aggregation arguments and $\operatorname{Sh}_{i}(N, \mu)$ be a Shapley weighting vector, then function $f$ is strictly monotonically increasing. Construct a penalty function $H$ as follows:

$$
H=\sum_{i=1}^{n} \operatorname{Sh}_{i}(N, \mu)\left[\frac{\lambda f\left(a_{i}\right)+(1-\lambda) f(y)}{\left(f\left(a_{i}\right)\right)^{\lambda}(f(y))^{1-\lambda}}-1\right],
$$

where $\lambda$ is a parameter that satisfies $\lambda \in[0,1]$. According to the necessary conditions for the existence of extreme values, taking the partial derivative of $H$ with respect to $y$, there is

$$
\frac{\partial H}{\partial y}=\sum_{i=1}^{n} \operatorname{Sh}_{i}(N, \mu)\left[\frac{(1-\lambda) f^{\prime}(y)\left(f\left(a_{i}\right)\right)^{\lambda}(f(y))^{1-\lambda}-\left[\lambda f\left(a_{i}\right)+(1-\lambda) f(y)\right]\left(f\left(a_{i}\right)\right)^{\lambda}(1-\lambda)(f(y))^{1-\lambda} f^{\prime}(y)}{\left(f\left(a_{i}\right)\right)^{2 \lambda}(f(y))^{2-2 \lambda}}\right] .
$$

Setting $(\partial H / \partial y)=0$, it follows that

$$
\sum_{i=1}^{n} \operatorname{Sh}_{i}(N, \mu)\left[\frac{\left(f\left(a_{i}\right)\right)^{\lambda} f(y) f^{\prime}(y)-\left[\lambda f\left(a_{i}\right)+(1-\lambda) f(y)\right]\left(f\left(a_{i}\right)\right)^{\lambda} f^{\prime}(y)}{\left(f\left(a_{i}\right)\right)^{2 \lambda}}\right]=0 .
$$

Equation (13) can be written as follows:

$$
\sum_{i=1}^{n} \operatorname{Sh}_{i}(N, \mu)\left[\frac{f(y) f^{\prime}(y)-\lambda f\left(a_{i}\right) f^{\prime}(y)-(1-\lambda) f(y) f^{\prime}(y)}{\left(f\left(a_{i}\right)\right)^{\lambda}}\right]=0 .
$$

Equation (14) is transformed into the following formula:

$$
f(y)=\frac{\sum_{i=1}^{n} \operatorname{Sh}_{i}(N, \mu)\left(f\left(a_{i}\right)\right)^{1-\lambda}}{\sum_{i=1}^{n} \operatorname{Sh}_{i}(N, \mu)\left(f\left(a_{i}\right)\right)^{-\lambda}} .
$$

The function $f$ is strictly monotonically increasing and has a reversible property. Thus, its inverse function exists as follows:

$$
y=f^{-1}\left(\frac{\sum_{i=1}^{n} \operatorname{Sh}_{i}(N, \mu)\left(f\left(a_{i}\right)\right)^{1-\lambda}}{\sum_{i=1}^{n} \operatorname{Sh}_{i}(N, \mu)\left(f\left(a_{i}\right)\right)^{-\lambda}}\right),
$$

and we called equation (16) as the Young-Shapley optimal weight (Y-SOW) operator.

In order to simplify the structure of the Y-SOW operator, the following equivalent expression is introduced in detail.

$$
\widehat{B}_{i}=\widehat{B}_{i}\left(a_{1}, \ldots, a_{n}\right)=\frac{\operatorname{Sh}_{i}(N, \mu)\left(f\left(a_{i}\right)\right)^{-\lambda}}{\sum_{i=1}^{n} \operatorname{Sh}_{i}(N, \mu)\left(f\left(a_{i}\right)\right)^{-\lambda}},
$$

then, it holds that $\sum_{i=1}^{n} \widehat{B}_{i}=1$. The Y-SOW operator can be equivalently written as

$$
\mathrm{Y}-\operatorname{SOW}\left(a_{1}, \ldots, a_{n}\right)=f^{-1}\left(\sum_{i=1}^{n} \widehat{B}_{i} f\left(a_{i}\right)\right) .
$$

The following theorem shows the monotonicity of $\widehat{B}_{i}$ with respect to $a_{i}$.

Theorem 1. Let $\lambda \in[0,1]$, then $\widehat{B}_{i}$ is monotonically decreasing with respect to $a_{i}$.

Proof. Taking the derivative of $\widehat{B}_{i}$ with respect to $a_{i}$, we have Let

$$
\frac{\partial \widehat{B}_{i}}{\partial a_{i}}=\frac{(-\lambda) \operatorname{Sh}_{i}(N, \mu)\left(f\left(a_{i}\right)\right)^{-\lambda-1} f^{\prime}\left(a_{i}\right) \sum_{i \neq j} \operatorname{Sh}_{i}(N, \mu)\left(f\left(a_{i}\right)\right)^{-\lambda}}{\left(\sum_{i=1}^{n} \operatorname{Sh}_{i}(N, \mu)\left(f\left(a_{i}\right)\right)^{-\lambda}\right)^{2}} .
$$


Since the function $f$ is monotonically increasing, we get $f^{\prime}\left(a_{i}\right) \geq 0$. Moreover, function $f$ is nonnegative and $\operatorname{Sh}_{i}(N, \mu) \geq 0,0 \leq \lambda \leq 1$, then we have

$$
\frac{\partial \widehat{B}_{i}}{\partial a_{i}} \leq 0
$$

Then, $\widehat{B}_{i}$ is monotonically decreasing.

Theorem 2. Let $a_{i}^{\prime}$ and $a_{i}(i=1,2, \ldots, n)$ be real numbers, then we have

(i) If $a_{i}^{\prime} \geq a_{i}$, then $\widehat{B}_{i}^{\prime}=\widehat{B}_{i}^{\prime}\left(a_{1}^{\prime}, \ldots, a_{i}^{\prime}, \ldots, a_{n}^{\prime}\right) \leq \widehat{B}_{i}\left(a_{1}, \ldots\right.$, $\left.a_{i}, \ldots, a_{n}\right)=\widehat{B}_{i}$

(ii) If $a_{i}^{\prime} \leq a_{i}$, then $\widehat{B}_{i}^{\prime}=\widehat{B}_{i}^{\prime}\left(a_{1}^{\prime}, \ldots, a_{i}^{\prime}, \ldots, a_{n}^{\prime}\right) \geq \widehat{B}_{i}\left(a_{1}, \ldots\right.$, $\left.a_{i}, \ldots, a_{n}\right)=\widehat{B}_{i}$

Proof. It can be known from Theorem 1, so the proof process is omitted.

3.2. Some Special Cases of the Y-SOW Operator. It is worth noting that the Y-SOW operator includes some special operators when the value of $\lambda$ is different or the expression of function $f(x)$ changes.

(i) When the weights of players (attributes) are indifferent and independent and $\operatorname{Sh}(N, \mu)=((1 / n)$, $(1 / n), \ldots,(1 / n))$ is selected, we have

$$
\mathrm{Y}-\operatorname{SOW}\left(a_{1}, \ldots, a_{n}\right)=f^{-1}\left(\frac{\sum_{i=1}^{n}\left(f\left(a_{i}\right)\right)^{1-\lambda}}{\sum_{i=1}^{n}\left(f\left(a_{i}\right)\right)^{-\lambda}}\right),
$$

then equation (21) becomes the Young-optimal weight (Y-OW) operator.

(ii) When $\lambda=1$, then

$$
\begin{aligned}
\mathrm{Y} & -\operatorname{SOW}\left(a_{1}, \ldots, a_{n}\right) \\
& =f^{-1}\left(\frac{1}{\sum_{i=1}^{n} \operatorname{Sh}_{i}(N, \mu)\left(1 /\left(f\left(a_{i}\right)\right)\right)}\right) .
\end{aligned}
$$

The Y-SOW operator reduces to the Shapley weighted harmonic averaging (SWHA) operator.

(iii) When $\lambda=0$, then

$\mathrm{Y}-\operatorname{SOW}\left(a_{1}, \ldots, a_{n}\right)=f^{-1}\left(\sum_{i=1}^{n} \operatorname{Sh}_{i}(N, \mu) f\left(a_{i}\right)\right)$.

Equation (23) reduces to quasi-Shapley weighted averaging (QSWA) operator.

(iv) When $f(x)=x$ is selected according to the overall risk attitude, the Y-SOW operator reduces to the following operator with respect to risk neutrality:

$$
\mathrm{Y}-\operatorname{SOW}\left(a_{1}, \ldots, a_{n}\right)=\frac{\sum_{i=1}^{n} \operatorname{Sh}_{i}(N, \mu)\left(a_{i}\right)^{1-\lambda}}{\sum_{i=1}^{n} \operatorname{Sh}_{i}(N, \mu)\left(a_{i}\right)^{-\lambda}} .
$$

(v) When $f(x)=\ln x$ is selected according to the overall risk attitude, the Y-SOW operator is simplified to the following operator in terms of risk aversion:

$$
\begin{aligned}
\mathrm{Y}- & \operatorname{SOW}\left(a_{1}, \ldots, a_{n}\right) \\
& =e^{\left(\sum_{i=1}^{n} \mathrm{Sh}_{i}(N, \mu)\left(\ln \left(a_{i}\right)\right)^{1-\lambda} / \sum_{i=1}^{n} \mathrm{Sh}_{i}(N, \mu)\left(\ln \left(a_{i}\right)\right)^{-\lambda}\right) .}
\end{aligned}
$$

If $\lambda=0$, equation (25) is changed to

$$
\begin{aligned}
& \mathrm{Y}-\operatorname{SOW}\left(a_{1}, \ldots, a_{n}\right)=e^{\left(\sum_{i=1}^{n} \mathrm{Sh}_{i}(N, \mu)\left(\ln \left(a_{i}\right)\right)^{1-\lambda} / \sum_{i=1}^{n} \mathrm{Sh}_{i}(N, \mu)\left(\ln \left(a_{i}\right)\right)^{-\lambda}\right)}
\end{aligned}
$$

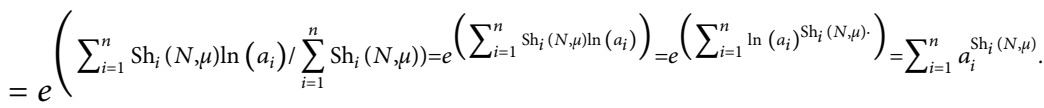

Equation (26) becomes the Shapley weighted geometric average (SWGA) operator.

(vi) When $f(x)=e^{x}$ is selected according to the decision makers overall risk attitude, the Y-SOW operator is reduced to the following operator in terms of risk proneness:

$\mathrm{Y}-\operatorname{SOW}\left(a_{1}, \ldots, a_{n}\right)=\ln \left(\frac{\sum_{i=1}^{n} \operatorname{Sh}_{i}(N, \mu) \cdot e^{(1-\lambda) \cdot a_{i}}}{\sum_{i=1}^{n} \operatorname{Sh}_{i}(N, \mu) \cdot e^{(-\lambda) \cdot a_{i}}}\right)$.
3.3. Desirable Property of the Y-SOW Operator. The Y-SOW operator has many potential properties, such as monotonicity, idempotency, and boundedness.

Property 1 (monotonicity). $\forall a_{i}^{\prime}, a_{i} \in R, \quad(i=1, \ldots, n)$. If $a_{i}^{\prime} \geq a_{i}$, it holds that

$$
\mathrm{Y}-\operatorname{SOW}\left(a_{1}^{\prime}, \ldots, a_{n}^{\prime}\right) \geq \mathrm{Y}-\operatorname{SOW}\left(a_{1}, \ldots, a_{n}\right) \text {. }
$$




$$
H=f^{-1}\left(\frac{\sum_{i=1}^{n} \operatorname{Sh}_{i}(N, \mu)\left(f\left(a_{i}\right)\right)^{1-\lambda}}{\sum_{i=1}^{n} \operatorname{Sh}_{i}(N, \mu)\left(f\left(a_{i}\right)\right)^{-\lambda}}\right) .
$$

Taking the derivative of the $\ln f(H)$ with respect to $a_{i}(i=1,2, \ldots, n)$, respectively, we get

It follows that

$\ln f(H)=\ln \sum_{i=1}^{n} \operatorname{Sh}_{i}(N, \mu)\left(f\left(a_{i}\right)\right)^{1-\lambda}-\ln \sum_{i=1}^{n} \operatorname{Sh}_{i}(N, \mu)\left(f\left(a_{i}\right)\right)^{-\lambda}$.

$$
\begin{aligned}
\frac{\partial(\ln f(H))}{\partial a_{i}} & =\frac{\operatorname{Sh}_{i}(N, \mu) \cdot(1-\lambda) \cdot\left(f\left(a_{i}\right)\right)^{-\lambda} \cdot f^{\prime}\left(a_{i}\right)}{\sum_{i=1}^{n} \operatorname{Sh}_{i}(N, \mu)\left(f\left(a_{i}\right)\right)^{1-\lambda}}-\frac{\operatorname{Sh}_{i}(N, \mu) \cdot(-\lambda) \cdot\left(f\left(a_{i}\right)\right)^{-\lambda-1} \cdot f^{\prime}\left(a_{i}\right)}{\sum_{i=1}^{n} \operatorname{Sh}_{i}(N, \mu)\left(f\left(a_{i}\right)\right)^{-\lambda}} \\
& =\operatorname{Sh}_{i}(N, \mu) \cdot f^{\prime}\left(a_{i}\right) \cdot\left(\frac{(1-\lambda) \cdot\left(f\left(a_{i}\right)\right)^{-\lambda}}{\sum_{i=1}^{n} \operatorname{Sh}_{i}(N, \mu)\left(f\left(a_{i}\right)\right)^{1-\lambda}}+\frac{\lambda \cdot\left(f\left(a_{i}\right)\right)^{-\lambda-1}}{\sum_{i=1}^{n} \operatorname{Sh}_{i}(N, \mu)\left(f\left(a_{i}\right)\right)^{-\lambda}}\right) .
\end{aligned}
$$

Since $f:[1, T] \longrightarrow[0,+\infty]$ and $f$ is increasing and nonnegative, we have $f^{\prime}\left(a_{i}\right) \geq 0$. According to Definition 7, we have $\operatorname{Sh}_{i}(N, \mu) \geq 0, \lambda \in[0,1]$. Therefore, $(\partial(\ln f(H))$ $\left(\partial a_{i}\right) \geq 0$. This means that $\ln f(H)$ is a monotonically increasing function. Obviously, $f(H)$ is also a monotonically increasing function. Thus, we get

$$
\frac{\sum_{i=1}^{n} \operatorname{Sh}_{i}(N, \mu)\left(f\left(a_{i}^{\prime}\right)\right)^{1-\lambda}}{\sum_{i=1}^{n} \operatorname{Sh}_{i}(N, \mu)\left(f\left(a_{i}^{\prime}\right)\right)^{-\lambda}} \geq \frac{\sum_{i=1}^{n} \operatorname{Sh}_{i}(N, \mu)\left(f\left(a_{i}\right)\right)^{1-\lambda}}{\sum_{i=1}^{n} \operatorname{Sh}_{i}(N, \mu)\left(f\left(a_{i}\right)\right)^{-\lambda}},
$$

which can be equivalently expressed as

$$
\begin{aligned}
& f^{-1}\left(\frac{\sum_{i=1}^{n} \operatorname{Sh}_{i}(N, \mu)\left(f\left(a_{i}^{\prime}\right)\right)^{1-\lambda}}{\sum_{i=1}^{n} \operatorname{Sh}_{i}(N, \mu)\left(f\left(a_{i}^{\prime}\right)\right)^{-\lambda}}\right) \\
& \quad \geq f^{-1}\left(\frac{\sum_{i=1}^{n} \operatorname{Sh}_{i}(N, \mu)\left(f\left(a_{i}\right)\right)^{1-\lambda}}{\sum_{i=1}^{n} \operatorname{Sh}_{i}(N, \mu)\left(f\left(a_{i}\right)\right)^{-\lambda}}\right) .
\end{aligned}
$$

To sum up, we have $f\left(a_{1}^{\prime}, \ldots, a_{n}^{\prime}\right) \geq f\left(a_{1}, \ldots, a_{n}\right)$.

Therefore, Property 1 is proved.

Property 2 (idempotency). $a, a_{i} \in R,(i=1, \ldots, n)$. If $a_{i}=a$ for all $i \in\{1, \ldots, n\}$, then

$$
\mathrm{Y}-\operatorname{SOW}\left(a_{1}, \ldots, a_{n}\right)=a \text {. }
$$

Proof:. Denoting

$$
\mathrm{Y}-\operatorname{SOW}\left(a_{1}, \ldots, a_{n}\right)=f^{-1}\left(\frac{\sum_{i=1}^{n} \operatorname{Sh}_{i}(N, \mu)\left(f\left(a_{i}\right)\right)^{1-\lambda}}{\sum_{i=1}^{n} \operatorname{Sh}_{i}(N, \mu)\left(f\left(a_{i}\right)\right)^{-\lambda}}\right) .
$$

Since $a_{i}=a$, for all $i=1, \ldots, n$, we have

$$
\begin{aligned}
\mathrm{Y}-\operatorname{SOW}\left(a_{1}, \ldots, a_{n}\right) & =f^{-1}\left(\frac{\sum_{i=1}^{n} \operatorname{Sh}_{i}(N, \mu)\left(f\left(a_{i}\right)\right)^{1-\lambda}}{\sum_{i=1}^{n} \operatorname{Sh}_{i}(N, \mu)\left(f\left(a_{i}\right)\right)^{-\lambda}}\right) \\
& =f^{-1}\left(\frac{(f(a))^{1-\lambda} \sum_{i=1}^{n} \operatorname{Sh}_{i}(N, \mu)}{(f(a))^{-\lambda} \sum_{i=1}^{n} \operatorname{Sh}_{i}(N, \mu)}\right) \\
& =f^{-1}(f(a))=a .
\end{aligned}
$$

Therefore, Property 2 is proved.

Property 3 (boundedness). $a_{i} \in R(i=1, \ldots, n)$ and denoting $\max _{i} a_{i}=a_{\max }$ and $\min _{i} a_{i}=a_{\min }$, then

$$
a_{\min } \leq \mathrm{Y}^{i}-\operatorname{SOW}\left(a_{1}, \ldots, a_{n}\right) \leq a_{\max }
$$

Proof. Denoting

$$
\mathrm{Y}-\operatorname{SOW}\left(a_{1}, \ldots, a_{n}\right)=f^{-1}\left(\frac{\sum_{i=1}^{n} \operatorname{Sh}_{i}(N, \mu)\left(f\left(a_{i}\right)\right)^{1-\lambda}}{\sum_{i=1}^{n} \operatorname{Sh}_{i}(N, \mu)\left(f\left(a_{i}\right)\right)^{-\lambda}}\right) .
$$

By Theorem 1, we have

$$
\mathrm{Y}-\operatorname{SOW}\left(a_{1}, \ldots, a_{n}\right)=f^{-1}\left(\sum_{i=1}^{n} \widehat{B}_{i} f\left(a_{i}\right)\right) \text {. }
$$

Because $\lambda \in[0,1]$, we have

$$
\sum_{i=1}^{n} \widehat{B}_{i} f\left(a_{i}\right) \geq \sum_{i=1}^{n} \widehat{B}_{i} f\left(a_{\min }\right)=f\left(a_{\min }\right) \sum_{i=1}^{n} \widehat{B}_{i}=f\left(a_{\min }\right), \sum_{i=1}^{n} \widehat{B}_{i} f\left(a_{i}\right) \leq \sum_{i=1}^{n} \widehat{B}_{i} f\left(a_{\max }\right)=f\left(a_{\max }\right) \sum_{i=1}^{n} \widehat{B}_{i}=f\left(a_{\max }\right) .
$$


Thus, we get

$$
f\left(a_{\min }\right) \leq \sum_{i=1}^{n} \widehat{B}_{i} f\left(a_{i}\right) \leq f\left(a_{\max }\right) .
$$

It follows that

$$
a_{\min } \leq f^{-1}\left(\sum_{i=1}^{n} \widehat{B}_{i} f\left(a_{i}\right)\right) \leq a_{\max } .
$$

To sum up, we have

$$
a_{\min } \leq \mathrm{Y}-\operatorname{SOW}\left(a_{1}, \ldots, a_{n}\right) \leq a_{\max } .
$$

The proof of Property 3 is completed.

\section{Deriving the Optimal Weight Vector Based on Dispersion Maximization Method}

Aggregation operators play a vital role in multiple fields, such as economics, statistics, and management. In case that the attribute associated with weight information is unknown, we establish the optimal weight vector based on the dispersion maximization method.

Suppose $A=\left\{a_{1}, \ldots, a_{m}\right\}$ is a finite set of alternatives, $C=\left\{c_{1}, \ldots, c_{n}\right\}$ is a set of attributes and $D=\left\{d_{1}, \ldots, d_{t}\right\}$ is the set of decision makers and $v=\left(v_{1}, \ldots, v_{t}\right)^{T}$ is the weight vector of a decision maker, which satisfies the condition $v_{k} \in[0,1], \sum_{k=1}^{t} v_{k}=1$. Assume that $A^{(k)}=\left(a_{i j}^{(k)}\right)_{m \times n}$ is the decision matrix given by the decision maker $d_{k}$, where the estimated value $a_{i j}^{(k)}$ indicates that the alternative $a_{i} \in A$ under attributes $c_{j} \in C$ is given by the decision maker $d_{k}$. $\omega=\left(\omega_{1}, \omega_{2}, \cdots, \omega_{t}\right)^{T}$ is the weight of the decision maker, satisfying $\omega_{i} \in[0,1], \sum_{i=1}^{t} \omega_{i}=1$.

In multiattribute decision-making, the attributes cannot be aggregated directly due to their different size. The primitive decision maker matrix has to be normalized. The attributes mainly include three types: benefit attribute, cost attribute, and fixed attribute. The benefit attribute refers to the bigger the better index, the cost attribute refers to the smaller the better index, and the fixed attribute refers to how close is it to a fixed value.
Let $H_{i}(i=1,2,3)$ be the subscript set of the above three types of attributes in order [10], and the following formula can be used for normalization:

$$
\begin{aligned}
& r_{i j}=\frac{a_{i j}}{\min _{i \in N} a_{i j}}, \quad j \in H_{1}, \\
& r_{i j}=\frac{\max _{i \in N} a_{i j}}{a_{i j}}, \quad j \in H_{2}, \\
& r_{i j}=1-\frac{\left|a_{i j}-a_{j}\right|}{\max \left|a_{i j}-a_{j}\right|}, \quad j \in H_{3} .
\end{aligned}
$$

For multiattribute decision-making, if the attribute $u_{j}$ can make a bigger difference in the attribute values of all alternatives, it means that $u_{j}$ plays a bigger role in the ordering of the alternatives and shall be given a bigger weight, vise verse. In particular, if all of the alternatives have no difference in the attribute values under the attribute $u_{j}, u_{j}$ will have no effect on the ordering of the alternatives and $u_{j}$ is zero. Based on that the weight can be determined with the dispersion maximization method.

Because there is a certain interaction between the attributes, in order to simplify the complexity of the fuzzy measure, the Shapley value based on the 2-additive measure is introduced as the weight of the attributes and the traditional Shapley value function has been mentioned in equation (9).

When the fuzzy measure $\mu$ is a 2 -additive measure, equation (9) is converted to [36-38]

$$
\operatorname{Sh}_{i}(N, \mu)=\frac{3-n}{2} \mu(i)+\sum_{j \in N \backslash i} \frac{1}{2}(\mu(i, j)-\mu(j)), \quad \forall i \in N \text {. }
$$

In Definition $7, \mathrm{Sh}_{i}(N, \mu)$ can be regarded as a weight vector. In view of Definition 6 and equation (47), the dispersion maximization model based on the 2 -additive measure and Shapley value is established as follows:

Model $(M-1)$ :

$$
\begin{aligned}
& \max T=\frac{3-n}{2} \sum_{j=1}^{m} \sum_{i=1}^{n} \sum_{k=1}^{n}\left(r_{i j}-r_{k j}\right)^{2} \mu(j)+\frac{1}{2} \sum_{j=1}^{m} \sum_{i=1}^{n} \sum_{k=1}^{n}\left(r_{i j}-r_{k j}\right)^{2}\left(\sum_{l \in N \backslash j} \mu(j, l)-\mu(l)\right), \\
& \text { s.t. }\left\{\begin{array}{l}
\sum_{l \subseteq S \backslash j}(\mu(j, l))-\mu(l) \geq(s-2) \mu\left(c_{j}\right), \quad \forall S \subseteq N, \forall l \in S, s \geq 2, \\
\sum_{\{j, l\} \subseteq N} \mu(j, l)-(n-2) \sum_{j \in N} \mu(j)=1, \\
\mu(j) \geq 0, \quad j=1,2, \ldots, n .
\end{array}\right.
\end{aligned}
$$

Finally, the optimal 2-additive measure can be obtained, and the optimal 2-additive measure can be reused to calculate the Shapley value in the Y-SOW operator. Using equation (16) to aggregate the Y-SOW operator, the optimal result is obtained.

Therefore, the Y-SOW operator-based multiattribute group decision (YSMAGD) algorithm is stepped as follows:
Step 1. Assuming that $A^{(k)}=\left(a_{i j}^{(k)}\right)_{m \times n}$ is the decision matrix given by the decision maker $d_{k}(k=1,2, \ldots, t)$.

Step 2. By equations (2) and (3), according to the decision makers' preference, the fuzzy linguistic quantifier with the pair $(a, b)$ is selected to compute the 


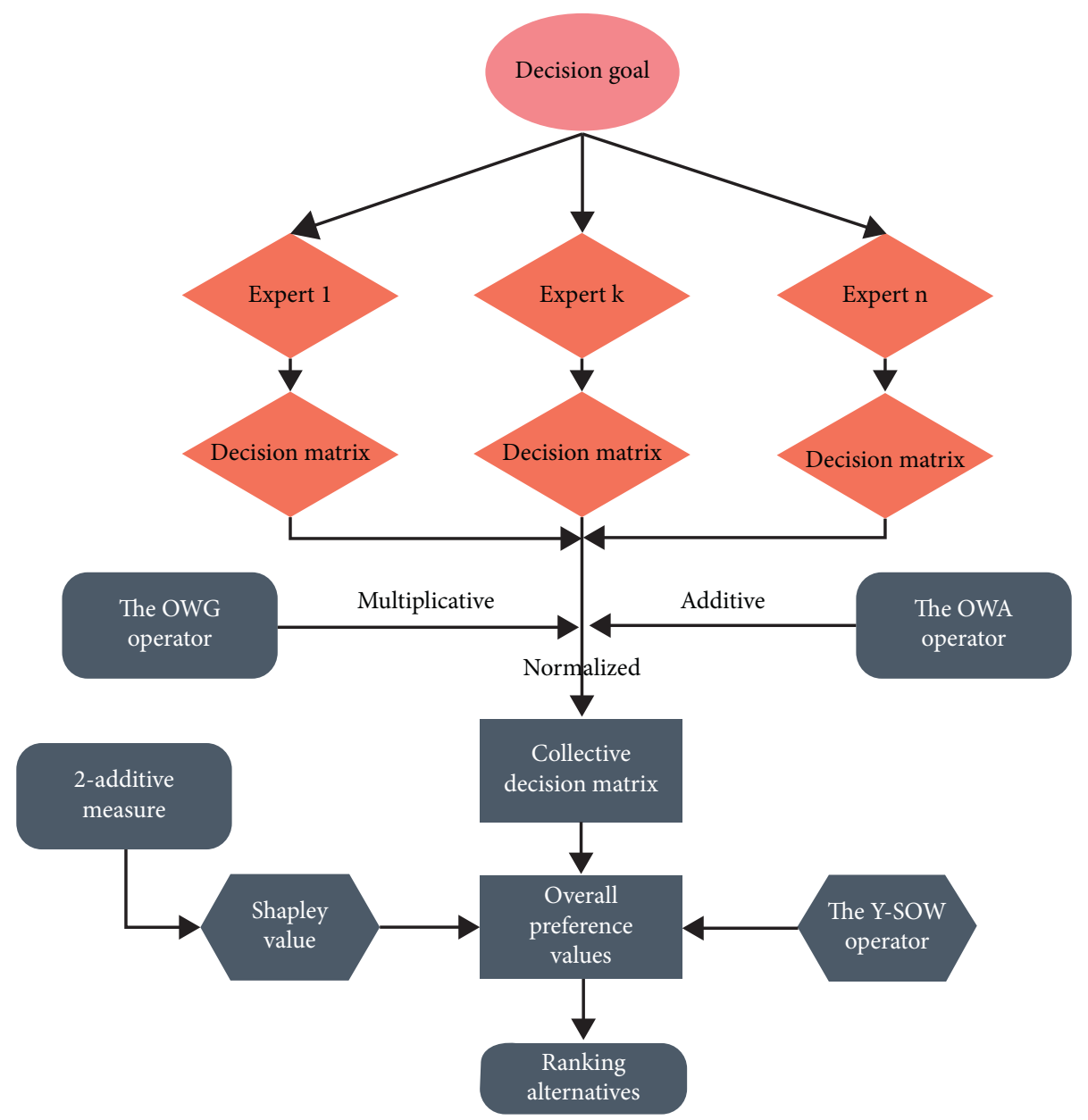

FIGURE 1: Framework of the YSMAGD algorithm.

weights of the decision makers $w_{j}=\left(w_{1}, w_{2}, \ldots, w_{n}\right)^{T}$ $(j=1,2, \ldots, n)$.

Step 3. Based on equations (1) and (4), the OWA operator and the OWG operator are used to aggregate the additive and multiplicative information of the decision matrix $A^{(k)}$, respectively. Final decision matrix $\bar{A}=\left(\bar{r}_{i j}\right)_{m \times n}$ is obtained.

Step 4. The final decision matrix $\bar{A}$ is normalized by equations (44)-(46) to obtain a collective decision matrix $R$.

Step 5. Utilize the dispersion maximization model $(M-1)$ to obtain the optimal 2-additive measure $\mu(i)$ and $\mu(i, j)$.

Step 6. $\mu(i)$ and $\mu(i, j)$ are used to calculate the Shapley value based on equation (47), that is, the weight vector of the Y-SOW operator.

Step 7. Utilize equation (16) to aggregate the normalized matrix $R$, and the overall preference value $\overline{r_{i}}(r=1, \ldots, m)$ of the alternative $a_{i}$ is obtained.

Step 8. Rank all the alternatives $a_{i}(i=1, \ldots, m)$ and select the best one in accordance with the ranking of $\bar{r}_{i}(r=1, \ldots, m)$.

Step 9. End.

Based on the above analysis, the framework of the YSMAGD algorithm is illustrated in Figure 1.

\section{Numerical Example}

The economic development brings us livelihood improvement, but with increasing land pollution. It is investigated that among the total 150 million mu of cultivated land in the country, 32.5 million $\mathrm{mu}$ is contaminated by sewage, 2 million $\mathrm{mu}$ is occupied by solid waste, and 2 million mu is destroyed, accounting for over $20 \%$. Crops accumulate harmful substances from polluted land, which causes diseases and ultimately endanger human future. However, the prevention and control is still weak. Nowadays, distribution and extent of soil pollution in the country are unclear. As a result, the government lacks specific control measures and capital input, and experts on land science research are also difficult to carry out in depth.

The first task is to select the polluted location for remediation. There are many both objective and subjective factors involved. The evaluation index is usually chaotic and miscellaneous. It is a typical problem of multiattribute aggregation with additive and multiplicative information. The proposed Y-SOW operator above is applied to decision analysis.

Consider site selection for land remediation, that is, the assessment of polluted land. Assume that there are eight land experts $\left(d_{1}, d_{2}, \ldots, d_{8}\right)$, evaluating the improvement of rural land from six attributes: $c_{1}$ pollution area, $c_{2}$ remediation potential, $c_{3}$ realistic feasibility, $c_{4}$ fertilizer and pesticide use rate, $c_{5}$ 
TABLE 1: 1-9 comparison scale.

\begin{tabular}{lc}
\hline Scale value & Meaning \\
\hline 1 & When $F_{i}$ is as important as $F_{j}$ \\
3 & When $F_{i}$ is slightly more important than $F_{j}$ \\
5 & When $F_{i}$ is more important than $F_{j}$ \\
7 & When $F_{i}$ is much more important than $F_{j}$ \\
9 & When $F_{i}$ is extremely important than $F_{j}$ \\
$2,4,6,8$ & A compromise of the above degree \\
\hline
\end{tabular}

soil $\mathrm{pH}$, and $c_{6}$ irrigation guarantee rate. There exist four lands $(A, B, C, D)$, and eight experts score four lands according to the above six attributes.

Step 1. Because the analytic hierarchy process (AHP) [39-41] can easily and flexibly deal with the quantitative problem of decision makers on complex systems, it is a classic weighting method commonly used by decision makers. The core of the method is to compare the degree of importance between the two elements by the 1 to 9 scale method. $c_{2}$ and $c_{3}$ are multiplicative indicators and cannot be calculated by specific numerical values. Therefore, the AHP scale method is used. Suppose there is a sample land $E$ with a scale of 1 for each attribute. Let land $E$ be $F_{j}$ and lands $A B C D$ be $F_{i}$. The experts compare the four lands $A B C D$ with the sample land $E$ according to the following 1-9 scale (shown in Table 1).

Let us suppose that the decision matrix $A^{(k)}=\left(a_{i j}^{(k)}\right)_{4 \times 6}$ provided by decision makers $d_{k}(k=1,2, \ldots, 8)$ is obtained as follows:

$$
\begin{aligned}
& A^{(1)}=\left(\begin{array}{cccccc}
40 & 7 & 6 & 0.6 & 5 & 0.5 \\
60 & 4 & 5 & 0.7 & 5.5 & 0.3 \\
55 & 5 & 6 & 0.5 & 7 & 0.7 \\
70 & 3 & 4 & 0.6 & 8.5 & 0.4
\end{array}\right) \text {, } \\
& A^{(2)}=\left(\begin{array}{llllll}
43 & 6 & 7 & 0.4 & 4.8 & 0.4 \\
58 & 5 & 4 & 0.8 & 5.8 & 0.7 \\
48 & 7 & 6 & 0.4 & 7.5 & 0.8 \\
65 & 4 & 3 & 0.7 & 8 & 0.4
\end{array}\right) \text {, } \\
& A^{(3)}=\left(\begin{array}{cccccc}
48 & 6 & 7 & 0.3 & 5.4 & 0.7 \\
62 & 5 & 5 & 0.7 & 6 & 0.5 \\
53 & 7 & 7 & 0.6 & 7.8 & 0.8 \\
76 & 4 & 4 & 0.7 & 8.7 & 0.4
\end{array}\right) \text {, } \\
& A^{(4)}=\left(\begin{array}{cccccc}
38 & 8 & 7 & 0.3 & 5.8 & 0.6 \\
58 & 6 & 6 & 0.6 & 6.6 & 0.7 \\
50 & 7 & 7 & 0.5 & 7 & 0.7 \\
80 & 4 & 3 & 0.8 & 8 & 0.2
\end{array}\right) \text {, } \\
& A^{(5)}=\left(\begin{array}{cccccc}
45 & 6 & 7 & 0.5 & 5.5 & 0.6 \\
55 & 5 & 5 & 0.6 & 5 & 0.4 \\
50 & 5 & 7 & 0.5 & 7.4 & 0.7 \\
73 & 4 & 3 & 0.7 & 8.8 & 0.3
\end{array}\right) \text {, }
\end{aligned}
$$

$$
\begin{aligned}
A^{(6)} & =\left(\begin{array}{llllll}
40 & 6 & 7 & 0.4 & 5.3 & 0.7 \\
58 & 4 & 5 & 0.6 & 5.5 & 0.5 \\
58 & 5 & 7 & 0.2 & 7.2 & 0.8 \\
66 & 3 & 3 & 0.8 & 8.2 & 0.4
\end{array}\right), \\
A^{(7)} & =\left(\begin{array}{llllll}
45 & 6 & 7 & 0.5 & 5.3 & 0.7 \\
62 & 3 & 5 & 0.7 & 5 & 0.4 \\
52 & 5 & 7 & 0.4 & 7.5 & 0.7 \\
71 & 2 & 2 & 0.7 & 9 & 0.3
\end{array}\right), \\
A^{(8)} & =\left(\begin{array}{llllll}
47 & 6 & 6 & 0.6 & 5.3 & 0.6 \\
65 & 3 & 3 & 0.8 & 4.8 & 0.3 \\
50 & 5 & 7 & 0.5 & 7.2 & 0.7 \\
75 & 2 & 3 & 0.8 & 8.3 & 0.3
\end{array}\right) .
\end{aligned}
$$

Step 2. By equations (2) and (3), and according to the decision makers' preference, the fuzzy linguistic quantifier "more," with the pair $(0.3,0.8)$ is selected to compute all the weights of the decision makers $w_{j}=\left(w_{1}, w_{2}, \ldots, w_{n}\right)^{T}(j=1,2, \ldots, n)$.

$$
\begin{aligned}
& w_{1}=Q\left(\frac{1}{8}\right)-Q(0)=0, \\
& w_{2}=Q\left(\frac{1}{4}\right)-Q\left(\frac{1}{8}\right)=0, \\
& w_{3}=Q\left(\frac{3}{8}\right)-Q\left(\frac{1}{4}\right)=0.15, \\
& w_{4}=Q\left(\frac{1}{2}\right)-Q\left(\frac{3}{8}\right)=0.25, \\
& w_{5}=Q\left(\frac{5}{8}\right)-Q\left(\frac{1}{2}\right)=0.25, \\
& w_{6}=Q\left(\frac{3}{4}\right)-Q\left(\frac{5}{8}\right)=0.25, \\
& w_{7}=Q\left(\frac{7}{8}\right)-Q\left(\frac{3}{4}\right)=0.1, \\
& w_{8}=Q(1)-Q\left(\frac{7}{8}\right)=0 .
\end{aligned}
$$

Finally, we can derive the weights of the decision makers $w=(0,0,0.15,0.25,0.25,0.25,0.1,0)^{T}$.

Step 3. $c_{2}$ and $c_{3}$ are multiplicative indicators, and the rest are additive indicators, so it cannot be directly aggregated. Based on equations (1) and (4) to aggregate the additive and multiplicative of the decision matrix $A^{(k)}$, obtain $\bar{A} \lim _{x \longrightarrow \infty}=\left(\bar{r}_{i j}\right)_{m \times n}$ :

$\bar{A}=\left(\begin{array}{cccccc}42.4500 & 10.3165 & 10.4335 & 0.4250 & 5.4900 & 0.6500 \\ 58.2500 & 9.8638 & 10.0035 & 0.6250 & 5.6750 & 0.5150 \\ 52.6500 & 10.1308 & 10.4335 & 0.4300 & 7.3200 & 0.7400 \\ 73.2500 & 9.4474 & 9.2511 & 0.7500 & 8.4550 & 0.3150\end{array}\right)$.

Step 4. It is clear that $c_{2}, c_{3}$, and $c_{6}$ are benefit attributes, $c_{1}$ and $c_{4}$ are cost attributes, and $c_{5}$ is the fixed attribute. 
Equations (44)-(46) are used for normalization to obtain a collective decision matrix $\bar{R}$ :

$\bar{R}=\left(\begin{array}{llllll}1.0000 & 1.0000 & 1.0000 & 1.0000 & 0.0662 & 0.8784 \\ 0.7288 & 0.9561 & 0.9588 & 0.6800 & 0.1225 & 0.6959 \\ 0.8063 & 0.9820 & 0.9820 & 0.9884 & 0.7881 & 1.0000 \\ 0.5795 & 0.9158 & 0.8867 & 0.5667 & 0.0364 & 0.4257\end{array}\right)$.

(52)
Step 5. From the above model $(M-1)$, the dispersion maximization model based on the 2 -additive measure and Shapley value is established to obtain the optimal 2additive measure $\mu(i)$ and $\mu(i, j)$.

$$
\begin{aligned}
& \max (-3.28 \mu(1)-3.55 \mu(2)-4.26 \mu(3)-3.78 \mu(4)-4.54 \mu(5)-3.22 \mu(6)+0.38 \mu(1,2)+0.4 \mu(1,3) \\
& +0.94 \mu(1,4)+1.91 \mu(1,5)+1.12 \mu(1,6)+0.05 \mu(2,3)+0.59 \mu(2,4)+1.56 \mu(2,5)+0.76 \mu(2.6) \\
& +0.61 \mu(3,4)+1.57 \mu(3,5)+0.78 \mu(3,6)+2.12 \mu(4,5)+1.32 \mu(4,6)+2.23 \mu(5,6)), \\
& \text { s.t. }\left\{\begin{array}{l}
\sum_{l \subseteq S \backslash j}(\mu(j, l))-\mu(l) \geq(s-2) \mu(j) \forall S \subseteq\{1,2,3,4,5,6\}, \quad \forall l \in S, \quad s \geq 2, \\
\sum_{\{j, l\} \subseteq\{1,2,3,4,5,6\}} \mu(j, l)-4(\mu(1)+\mu(2)+\mu(3)+\mu(4)+\mu(5)+\mu(6))=1, \\
\mu(j) \geq 0, \quad j=1,2, \ldots, 6 .
\end{array}\right.
\end{aligned}
$$

Solving the above linear programming model $(M-1)$, we get

$$
\begin{aligned}
\mu(1) & =0.05, \\
\mu(2) & =0.1, \\
\mu(3) & =0.1, \\
\mu(4) & =0.1, \\
\mu(5) & =0.05, \\
\mu(6) & =0.05, \\
\mu(1,2) & =0.0081, \\
\mu(1,3) & =0.0091, \\
\mu(1,4) & =0.0013, \\
\mu(1,5) & =0.2252, \\
\mu(1,6) & =0.0063, \\
\mu(2,3) & =0.001, \\
\mu(2,4) & =0.0028, \\
\mu(2,5) & =0.2617, \\
\mu(2,6) & =0.0096, \\
\mu(3,4) & =0.0096, \\
\mu(3,5) & =0.2556, \\
\mu(3,6) & =0.0097, \\
\mu(4,5) & =0.2701, \\
\mu(4,6) & =0.0049, \\
\mu(5,6) & =0.3250 .
\end{aligned}
$$


These are the optimal 2-additive measures.

Step 6. From Equation (47), the Shapley value of the $\mathrm{Y}$-SOW operator is calculated as follows:

$$
\begin{aligned}
& \operatorname{Sh}_{1}(N, \mu)=-\frac{3}{2} \mu(1)+\frac{1}{2}[\mu(1,2)-\mu(2)+\mu(1,3)-\mu(3)+\mu(1,4)-\mu(4)+\mu(1,5)-\mu(5)+\mu(1,6)-\mu(6)]=0.16, \\
& \operatorname{Sh}_{2}(N, \mu)=-\frac{3}{2} \mu(2)+\frac{1}{2}[\mu(2,1)-\mu(1)+\mu(2,3)-\mu(3)+\mu(2,4)-\mu(4)+\mu(2,5)-\mu(5)+\mu(2,6)-\mu(6)]=0.076, \\
& \operatorname{Sh}_{4}(N, \mu)=-\frac{3}{2} \mu(4)+\frac{1}{2}[\mu(4,1)-\mu(1)+\mu(4,2)-\mu(2)+\mu(4,3)-\mu(4)+\mu(4,5)-\mu(5)+\mu(4,6)-\mu(6)]=0.229, \\
& \operatorname{Sh}_{3}(N, \mu)=-\frac{3}{2} \mu(3)+\frac{1}{2}[\mu(3,1)-\mu(1)+\mu(3,2)-\mu(2)+\mu(3,4)-\mu(4)+\mu(3,5)-\mu(5)+\mu(3,6)-\mu(6)]=0.142, \\
& \operatorname{Sh}_{5}(N, \mu)=-\frac{3}{2} \mu(5)+\frac{1}{2}[\mu(5,1)-\mu(1)+\mu(5,2)-\mu(2)+\mu(5,3)-\mu(3)+\mu(5,4)-\mu(4)+\mu(5,6)-\mu(6)]=0.272, \\
& \operatorname{Sh}_{6}(N, \mu)=-\frac{3}{2} \mu(6)+\frac{1}{2}[\mu(6,1)-\mu(1)+\mu(6,2)-\mu(2)+\mu(6,3)-\mu(3)+\mu(6,4)-\mu(4)+\mu(6,5)-\mu(5)]=0.121 .
\end{aligned}
$$

Step 7. Based on equation (16), for convenience, we set $f(x)=x, \lambda=1$, and the overall preference value $\bar{r}_{i}(r=$ $1, \ldots, m)$ of the alternative $a_{i}$ is obtained. Let $\bar{r}_{i}=Y-\operatorname{SOW}\left(a_{1}, \ldots, a_{n}\right)$. The overall preference values are obtained as

$$
\begin{aligned}
& \bar{r}_{1}=4.858, \\
& \bar{r}_{2}=3.247, \\
& \bar{r}_{3}=4.323, \\
& \bar{r}_{4}=8.679 .
\end{aligned}
$$

Step 8. The ranking is $\bar{r}_{4}>\bar{r}_{1}>\bar{r}_{3}>\bar{r}_{2}$.

Therefore, the best alternative is $a_{4}$. Namely, land $D$ is most needed for remediation.

In this example, we can see that the YSMAGD algorithm can efficiently handle heterogeneous data with the good aggregation. The evaluation index of site selection of polluted land in the above example usually contains objective factors and subjective factors. For example, indicators such as remedial potential and realistic feasibility are multiplicative indicators that cannot be calculated from specific values. Indicators such as pollution area and soil $\mathrm{pH}$ are additive indicators that can be obtained through clear numerical measurements. The key to land pollution assessment is to combine both indicators, so decision makers use the Y-SOW operator proposed above to aggregate and use the YSMAGD algorithm for effective land assessment. This is not only beneficial for the government to implement land pollution remediation, but also for experts to conduct land research.

\section{Comparison and Conclusion}

\subsection{Comparison and Analysis}

6.1.1. Comparative Analysis of Aggregation Results under Different Values of $\lambda$. Because $\lambda \in[0,1]$, there are many options of parameter $\lambda$, such as $\lambda=0, \lambda=(1 / 2)$, and $\lambda=1$. Comparing these three situations, the overall preference value is obtained shown as follows:

(i) When $\lambda=0$,

$$
\begin{aligned}
& \bar{r}_{1}=1.368, \\
& \bar{r}_{2}=1.667, \\
& \bar{r}_{3}=1.105, \\
& \bar{r}_{4}=2.222 .
\end{aligned}
$$

(ii) When $\lambda=(1 / 2)$,

$$
\begin{aligned}
& \bar{r}_{1}=2.269, \\
& \bar{r}_{2}=2.191, \\
& \bar{r}_{3}=1.113, \\
& \bar{r}_{4}=3.730 .
\end{aligned}
$$

Compare the above three cases and use the obtained overall preference values as a scatter plot, as shown in Figure 2.

Obviously, for $\lambda=0, \lambda=(1 / 2)$, and $\lambda=1$, the results of aggregation are not numerically identical. However, by comparing these results, the same alternative approach can be obtained, namely, land $D$ needs to be repaired the most urgently. 


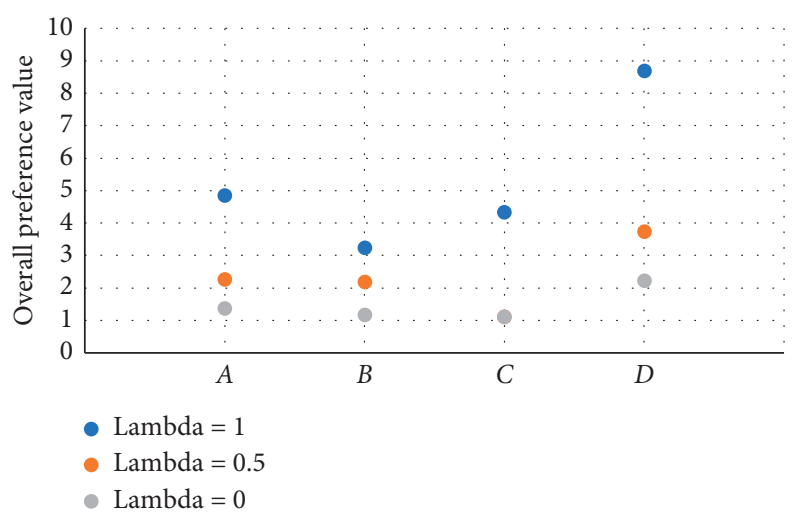

Figure 2: The overall preference values with respect to different $\lambda$.

6.1.2. Comparing the Y-SOW Operator with WAA Operator and WGA Operator. Let $a_{1}=(1 / 2), a_{2}=3$, and $a_{3}=8$ be three real numbers. For comparing with the weighted arithmetic averaging (WAA) operator [42] and weighted geometric averaging (WGA) operator [43], $\lambda=0.5$ and $f(x)=x$ are selected. Assume that decision makers (or attributes) are independent and unrelated, so we set weighted vector $w=\operatorname{Sh}(N, \mu)=((1 / 3),(1 / 3),(1 / 3))$. Accordingly, the aggregation results calculated by three aggregation operators are shown as follows:

$$
\begin{aligned}
\operatorname{WAA}\left(a_{1}, a_{2}, a_{3}\right) & =3.833, \\
\operatorname{WGA}\left(a_{1}, a_{2}, a_{3}\right) & =2.289, \\
\mathrm{Y}-\operatorname{SOW}\left(a_{1}, a_{2}, a_{3}\right) & =2.246 .
\end{aligned}
$$

We denote $H$ (WWA), $H$ (WGA), and $H(\mathrm{Y}-\mathrm{SOW})$ the penalty functions derived by equation (11), respectively. Thus, we have the following results:

$$
\begin{aligned}
H(\text { WWA }) & =0.214, \\
H(\text { WGA }) & =0.172, \\
H(\mathrm{Y}-\mathrm{SOW}) & =0.171 .
\end{aligned}
$$

It is clear that the value of penalty function $H$ with respect to the Y-SOW operator is the smallest. From equation (11), we know that the Y-SOW operator is the optimal aggregation for minimizing the penalty function. This supports the optimality of the Y-SOW operator.

6.2. Brief Conclusion. This paper introduces a new aggregation operator called the Young-Shapley optimal weight (Y-SOW) operator. Some special cases and main properties of the Y-SOW operator are studied. The advantages of this paper are as follows:

(i) The Y-SOW operator solves the problem that the additive and the multiplicative decision information appear simultaneously in the group decisionmaking.

(ii) In the Shapley value method of cooperative game, the 2-additive measure replaces the original fuzzy measure. This not only can effectively deal with the interaction between decision attributes, but also reduce the computational complexity and improve the representation between attributes.

(iii) To solve the problems of multiattribute group decision-making under attribute interaction, we develop the Y-SOW operator-based multiattribute group decision (YSMAGD) algorithm and establish a linear programming model $(M-1)$.

It is worth noting that we can also apply the Y-SOW operator to the other areas such as deep learning, cluster analysis, and artificial intelligence.

Nevertheless, in this article, we only consider the case where decision information is represented by real numbers. However, in some cases, the decision information may be in other forms, such as interval numbers and fuzzy numbers. This is an issue that needs further study in the near future.

\section{Data Availability}

The data used to support the findings of this study are included within the article.

\section{Conflicts of Interest}

The authors declare that they have no conflicts of interest.

\section{Acknowledgments}

This work was supported by the National Natural Science Foundation of China (nos. 71601049, 61972093, and 61501120), the Humanities and Social Sciences Fund of the Ministry of Education (no. 16YJC630064), the Foundation of Beijing Intelligent Logistics System Collaborative Innovation Center (no. BILSCIC-2019KF-16), China Postdoctoral Science Foundation (no. 2019M660242), the Construction Fund for Digital Fujian Big Data for Agriculture and Forestry (no. KJG18019A), and the University Training Program in Scientific Research for Outstanding Young Talents of Fujian Province: cost sharing strategy of incomplete cooperative games and its application in water pollution control (no. 117/K80SCC53A).

\section{References}

[1] C.-T. Chen, "Extensions of the TOPSIS for group decisionmaking under fuzzy environment," Fuzzy Sets and Systems, vol. 114 , no. 1 , pp. 1-9, 2000.

[2] F. Meng, Q. Zhang, and J. Zhan, "The interval-valued intuitionistic fuzzy geometric Choquet aggregation operator based on the generalized Banzhaf index and 2-additive measure," Technological and Economic Development of Economy, vol. 21, no. 2, pp. 186-215, 2015.

[3] P. Liu and D. Li, "Some muirhead mean operators for intuitionistic fuzzy numbers and their applications to group decision making," PLoS One, vol. 12, no. 1, Article ID e0168767, 2017.

[4] L. D. Miguel, M. Sesma-Sara, M. Elkano, M. Asiain, and H. Bustince, "An algorithm for group decision making using n-dimensional fuzzy sets, admissible orders and OWA operators," Information Fusion, vol. 37, pp. 126-131, 2017. 
[5] P. Rani, D. Jain, and D. S. Hooda, "Extension of intuitionistic fuzzy TODIM technique for multi-criteria decision making method based on Shapley weighted divergence measure," Granular Computing, vol. 4, no. 3, pp. 407-420, 2019.

[6] R. Yuan, J. Tang, and F. Meng, "Linguistic intuitionistic fuzzy group decision making based on aggregation operators," International Journal of Fuzzy Systems, vol. 21, no. 2, pp. 407-420, 2019.

[7] R. R. Yager, "On ordered weighted averaging aggregation operators in multicriteria decisionmaking," IEEE Transactions on Systems, Man, and Cybernetics, vol. 18, no. 1, pp. 183-190, 2002.

[8] Z. S. Xu and Q. L. Da, "The ordered weighted geometric averaging operators," International Journal of Intelligent Systems, vol. 17, no. 7, pp. 709-716, 2010.

[9] F. Chiclana, F. Herrera, and E. Herreraviedma, The Ordered Weighted Geometric Operator: Properties and Application, Springer, Berlin, Germany, 2000.

[10] H. Y. Chen and Z. C. Liu, "Induced ordered weighted harmonic averaging (IOWHA) operator and its application in combined prediction," Chinese Journal of Management Science, vol. 12, no. 5, pp. 35-40, 2004.

[11] R. R. Yager, "Generalized OWA aggregation operators," Fuzzy Optimization and Decision Making, vol. 3, no. 1, pp. 93-107, 2004.

[12] L. G. Zhou and H.-Y. Chen, "Generalized ordered weighted logarithm aggregation operators and their applications to group decision making," International Journal of Intelligent Systems, vol. 25, no. 7, pp. 683-707, 2010.

[13] L. Zhou, H. Chen, and J. Liu, "Generalized weighted exponential proportional aggregation operators and their applications to group decision making," Applied Mathematical Modelling, vol. 36, no. 9, pp. 4365-4384, 2012.

[14] J. M. Merigo, K. J. Engemann, and D. Palaciosmarques, "Decision making with Dempster-Shafer belief structure and the OWAWA operator," Technological \& Economic Development of Economy, vol. 19, no. 1, pp. S100-S118, 2014.

[15] L. G. Zhou and H. Y. Chen, "Continuous generalized OWA operator and its application to decision making," Fuzzy Sets and Systems, vol. 168, no. 1, pp. 18-34, 2011.

[16] G. Beliakov, S. James, J. Mordelová, T. Rückschlossová, and R. R. Yager, "Generalized Bonferroni mean operators in multi-criteria aggregation," Fuzzy Sets and Systems, vol. 161, no. 17, pp. 2227-2242, 2010.

[17] R. R. Yager, "Induced aggregation operators," Fuzzy Sets and Systems, vol. 137, no. 1, pp. 59-69, 2003.

[18] Z. Xu, "Dependent OWA operators," International Conference on Modeling Decisions for Artificial Intelligence, Springer, Berlin, Germany, 2006.

[19] Z. Xu and M. Xia, "Induced generalized intuitionistic fuzzy operators," Knowledge-Based Systems, vol. 24, no. 2, pp. 197-209, 2011.

[20] Z. Xu, "An overview of methods for determining owa weights," International Journal of Intelligent Systems, vol. 20, no. 8, pp. 843-865, 2005.

[21] R. R. Yager, J. Kacprzyk, and G. Beliakov, Recent Developments in the Ordered Weighted Averaging Operators: Theory and Practice, Springer, Berlin, Germany, 2011.

[22] M. Grabisch, "Fuzzy integral in multicriteria decision making," Fuzzy Sets and Systems, vol. 69, no. 3, pp. 279-298, 1995.

[23] M. Grabisch, "The application of fuzzy integrals in multicriteria decision making," European Journal of Operational Research, vol. 89, no. 3, pp. 445-456, 1996.
[24] M. Grabisch, T. Murofushi, and M. Sugeno, Fuzzy Measure and Integrals, Physica-Verlag, New York, NY, USA, 2000.

[25] M. Grabisch and C. Labreuche, "A decade of application of the Choquet and Sugeno integrals in multi-criteria decision aid," 4, vol. 6, no. 1, pp. 1-44, 2008.

[26] Z. Xu, "Choquet integrals of weighted intuitionistic fuzzy information," Information Sciences, vol. 180, no. 5, pp. 726736, 2010.

[27] T. Murofushi and M. Sugeno, "A theory of fuzzy measures: representations, the Choquet integral, and null sets," Journal of Mathematical Analysis and Applications, vol. 159, no. 2, pp. 532-549, 1991.

[28] R. R. Phelps, "Lectures on Choquet's theorem," Lecture Notes in Mathematics, p. 1757, Springer, Berlin, Germany, 2001.

[29] M. Sugeno, Theory of Fuzzy Integral and Its Application, Doctorial Dissertation, Tokyo Institute of Technology, Tokyo, Japan, 1974.

[30] M. Grabisch, " $k$-order additive discrete fuzzy measures and their representation," Fuzzy Sets and Systems, vol. 92, no. 2, pp. 167-189, 1997.

[31] L. G. Zhou, Several Kinds of Generalized Information Integration Operators and Their Application in Multi-Attribute Decision Making, Anhui University, Hefei, China, 2013.

[32] J. Liu, S. Lin, H. Chen, and L. Zhou, "The continuous quasiOWA operator and its application to group decision making," Group Decision and Negotiation, vol. 22, no. 4, pp. 715-738, 2013.

[33] R. R. Yager, "Families of OWA operators," Fuzzy Sets and Systems, vol. 59, no. 2, pp. 125-148, 1993.

[34] L. S. Shapley, "17. A value for n-person games," Contributions to the Theory of Games (AM-28), vol. 28, pp. 307-318, 1953.

[35] L. W. Yan, Typical Problems and Methods in Mathematical Analysis, Higher Education Press, Beijing, China, 2002.

[36] J. Lin, Q. Zhang, and F. Meng, "An approach for facility location selection based on optimal aggregation operator," Knowledge-Based Systems, vol. 85, pp. 143-158, 2015.

[37] F. Meng and X. Chen, The Symmetrical Interval Intuitionistic Uncertain Linguistic Operators and Their Application to Decision Making, Pergamon Press, Oxford, UK, 2016.

[38] J. Wu and Q. Zhang, "Multicriteria decision making method based on 2-order additive fuzzy measures," Systems Engineering-Theory \& Practice, vol. 30, no. 7, pp. 1229-1237, 2010.

[39] B. Özkan, O. Dengiz, and İ. D. Turan, "Site suitability assessment and mapping for rice cultivation using multi-criteria decision analysis based on fuzzy-AHP and TOPSIS approaches under semihumid ecological condition in delta plain," Paddy and Water Environment, vol. 174, no. 4, pp. 665-676, 2019.

[40] M. P. Amiri, "Project selection for oil-fields development by using the AHP and fuzzy TOPSIS methods," Expert Systems with Applications, vol. 37, no. 9, pp. 6218-6224, 2010.

[41] Y. J. Lu and W. Zhang, "The important role of index scale in the AHP scale system," Journal of Systems Engineering, vol. 18, no. 5, pp. 452-456, 2003.

[42] J. C. Harsanyi, "Cardinal welfare, individualistic ethics, and interpersonal comparisons of utility," Journal of Political Economy, vol. 63, no. 4, pp. 309-321, 1955.

[43] Z. S. Xu and Q. L. Da, "The ordered weighted geometric averaging operators," International Journal of Intelligent Systems, vol. 17, no. 7, pp. 709-716, 2002. 